Classification

Physics Abstracts

$07.50-35.80-34.50$

\title{
Générateur d'impulsions haute-tension à temps de montée rapide et à durée variable. Application à l'étude des niveaux de Rydberg
}

\author{
M. Ahrweiller, M. Hugon, P. R. Fournier et A. Hourdin \\ Service de physique des atomes et des surfaces, Centre d'Etudes Nucléaires de Saclay, \\ 91191 Gif sur Yvette Cedex, France
}

(Reçu le 2 novembre 1981, accepté le 18 janvier 1982)

\begin{abstract}
Résumé. - On présente un générateur haute-tension qui produit des impulsions rectangulaires d'amplitude variant de 0,1 à $3 \mathrm{kV}$ avec un taux de répétition pouvant atteindre $50 \mathrm{~Hz}$. Chaque impulsion a un temps de montée de 20 ns et une durée variable de 0,1 à $6 \mu$ s. Le faible « jitter » et la stabilité de ce générateur en font un instrument idéal pour faire des mesures à accumulation de données. On donne un exemple d'application de ce générateur à l'étude des niveaux de Rydberg.

Abstract. - We present a high-voltage generator which generates rectangular pulses having a variable amplitude from 0.1 to $3 \mathrm{kV}$ with a repetition rate up to $50 \mathrm{~Hz}$. The rise-time is $20 \mathrm{~ns}$ and the duration is variable from 0.1 to $6 \mu \mathrm{s}$ for each pulse. This generator is well adapted to make data-storage measurements because of its low jitter and its stability. An example is given for the application of this generator to the study of Rydberg levels.
\end{abstract}

1. Introduction. - Les atomes excités dans des niveaux de nombre quantique principal $n$ élevé (niveaux de Rydberg) font l'objet depuis quelques années d'études détaillées, en particulier dans notre laboratoire.

Une propriété importante des atomes excités dans des états de Rydberg est que l'électron excité a une faible énergie de liaison avec le cœur ionique $\left(W \sim 1 / n^{* 2}, n^{*}\right.$ étant le nombre quantique principal effectif de l'état de Rydberg). Ils peuvent donc être ionisés facilement par un champ électrique relativement faible $\left(E \simeq 2 \mathrm{kV} / \mathrm{cm}\right.$ pour $n^{*}=20$; ce champ $E$ varie en $\left.n^{*-4}\right)$. L'ionisation par champ électrique a une efficacité de l'ordre de $100 \%$ et constitue un moyen très sensible de détection des états de Rydberg élevés. Rappelons que leur détection par fluorescence a une efficacité qui décroît très vite avec $n$ et n'est utilisable que jusqu'à $n \simeq 20$. L'ionisation par champ électrique est, de plus, sélective, puisque des niveaux de Rydberg de $n$ différents s'ionisent à des valeurs différentes du champ électrique (en fait, ce processus est complexe, parce que le champ ionisant dépend aussi des autres nombres quantiques de l'état de Rydberg).

Afin d'étudier les propriétés collisionnelles d'atomes excités dans des états de Rydberg de $n \simeq 40$ [1], nous avons été amenés à réaliser un générateur d'impul- sions haute-tension d'un type nouveau. Son cahier des charges correspond à la technique expérimentale utilisée, qui est de mesurer la durée de vie d'un état de Rydberg à différentes pressions de gaz perturbateur. La variation de cette durée de vie permet de déduire la section efficace de dépopulation de l'état considéré par le gaz perturbateur. Dans l'expérience, les atomes sont excités dans un état de Rydberg donné entre deux plaques formant un condensateur plan (dipôle électrostatique) par un laser pulsé, dont la durée des pulses est d'environ $8 \mathrm{~ns}$. Le générateur branché aux bornes du dipôle électrostatique produit une impulsion de haute tension pour créer le champ électrique nécessaire à l'ionisation des atomes de Rydberg un certain temps $t(\sim 0,5$ à $60 \mu \mathrm{s})$ après l'excitation laser. Les ions formés sont accélérés par le champ électrique et la charge ionique mesurée est proportionnelle à la population de l'état de Rydberg à l'instant $t$. En faisant varier le retard entre l'excitation laser et l'impulsion de haute tension, on obtient l'évolution temporelle de cette population.

Le générateur d'impulsions haute-tension a les caractéristiques suivantes :

- amplitude : 0,1-3 $\mathrm{kV}$ permettant d'ioniser les niveaux de $n^{*}=24$ à 55 pour une distance entreplaques de $2,88 \mathrm{~cm}$; 
— temps de montée : 20 ns avec un « jitter » de 2 ns donnant une bonne résolution en temps $\left({ }^{1}\right)$;

- durée : 0,1-6 $\mu$ s permettant d'accélérer les ions formés ;

- taux de répétition : $2-50 \mathrm{~Hz}$ étant celui du laser pulsé ;

— retard : 10, 20 ou 30 pas de 0,2 à $2 \mu$ s permettant de mesurer les durées de vie, qui sont comprises typiquement entre 1,5 et $50 \mu \mathrm{s}$.

2. Description du générateur. - Nous avons conçu ce générateur de telle sorte que son impédance interne soit égale à celle de l'impédance du dipôle et à celle du câble coaxial de liaison. L'adaptation des impédances étant réalisée, on peut assurer cette liaison sur une distance de quelques mètres sans détérioration notable de la forme de l'impulsion. Ceci offre un grand avantage quant à l'accessibilité et à l'agencement de l'aire expérimentale. La capacité propre du dipôle étant de 15 picofarads, nous avons choisi le standard d'impédance de $100 \Omega$.

La variation nécessaire de la largeur $\tau_{1}$ de l'impulsion (de 0,1 à $6 \mu \mathrm{s}$ ) rend impossible la solution de générateur classique à ligne de retard (à constantes réparties ou localisées). Nous avons repris le principe d'un montage réalisé et mis au point au laboratoire [2], en améliorant ses performances, pour l'adapter à la nouvelle expérience.

Ce générateur comporte 3 parties : un étage de puissance, un étage d'attaque et une logique de commande.

2.1 Etage de PUisSANCE. - La figure 1 représente le schéma de principe de l'étage de puissance du générateur. La résistance de charge $R_{\mathrm{ch}}$ branchée en paral-

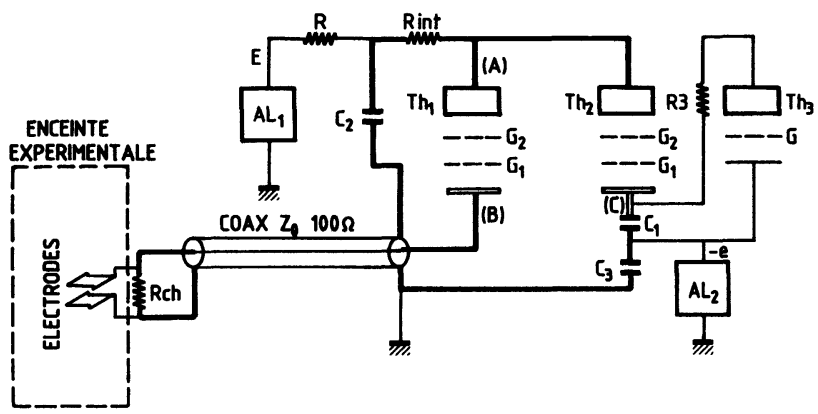

Fig. 1. - Schéma de principe de l'étage de puissance.

[Schematic diagram of the power stage.]

lèle sur le dipôle permet de respecter la relation $R_{\text {int }}=Z_{0}=R_{\mathrm{ch}}$. Cette résistance $R_{\mathrm{ch}}$ est placée au plus près de l'enceinte expérimentale avec des

(1) Il est aussi possible de faire varier le temps de montée de l'impulsion entre $20 \mathrm{~ns}$ et $5 \mu \mathrm{s}$. L'ionisation par champ électrique est un processus dynamique, et les résultats observés (champ ionisant, forme des raies) peuvent varier notablement avec ce temps de montée. connexions courtes pour réduire les capacités et les inductances parasites.

La partie du circuit représentée en trait gras a été réalisée en structure coaxiale. Cette précaution permet de diminuer des boucles parasites dont l'impédance aurait détérioré les temps de montée et de descente $\theta_{1}$ et $\theta_{2}$ et qui auraient été le siège de rayonnements électromagnétiques parasites importants.

Pour la commutation, le choix s'est porté sur les thyratrons tétrodes céramique du type CX1157 $\left(\mathrm{Th}_{1}\right.$, $\mathrm{Th}_{2}$ ). Ces thyratrons sont plus performants que les thyratrons triodes classiques. Ils ont un temps de "jitter ", un temps de délai et une dérive au départ de l'impulsion d'anode plus faibles et leur temps de montée est plus rapide en intensité [3], dans le cas présent le $\mathrm{d} i / \mathrm{d} t$ peut atteindre $10^{3} \mathrm{~A} / \mu \mathrm{s}$. Le $3^{\mathrm{e}}$ thyratron $\left(\mathrm{Th}_{3}\right)$ est un tube en verre classique du type $4 \mathrm{C} 35$, ses performances n'intervenant pas directement dans la forme de l'amplitude.

Le chauffage des réservoirs et des filaments des thyratrons $\left(\mathrm{Th}_{1}, \mathrm{Th}_{2}, \mathrm{Th}_{3}\right)$ est réglé par des transformateurs variables dans leur zone de fonctionnement optimale quelle que soit la valeur de l'amplitude de l'impulsion demandée.

La capacité $C_{2}$ assure le découplage de l'alimentation variable $\mathrm{AL}_{1}$ de tension $E$ et représente une impédance nulle en régime impulsionnel.

La capacité $C_{3}$ assure le découplage de l'alimentation fixe $\mathrm{AL}_{2}$ qui délivre la tension de polarisation $-e$. $R_{\text {int }}$ et $\boldsymbol{R}_{\mathrm{ch}}$ sont respectivement les résistances interne et de charge du générateur.

La figure 2 représente le diagramme de fonctionnement de l'étage de puissance.

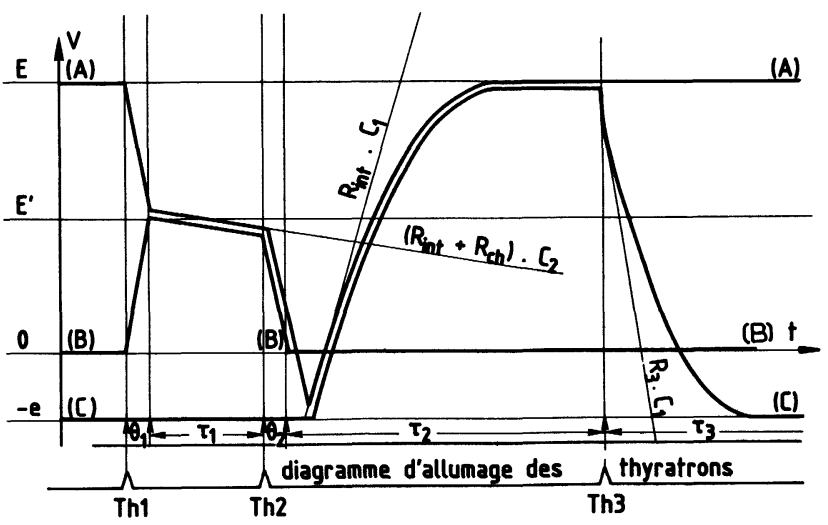

Fig. 2. - Diagramme de fonctionnement de l'étage de puissance.

[Working diagram of the power stage.]

Le cycle complet de formation d'une impulsion est effectué lorsque $\mathrm{Th}_{1}, \mathrm{Th}_{2}, \mathrm{Th}_{3}$ sont successivement allumés, éteints et désionisés.

Lorsque $\mathrm{Th}_{1}$ est allumé, le potentiel de cathode (B) passe de zéro à la valeur $E^{\prime}$ définie par le rapport potentiométrique de $R_{\mathrm{int}}$ et $\boldsymbol{R}_{\mathrm{ch}}$. En négligeant la tension d'arc de $\mathrm{Th}_{1}$, on a $E^{\prime}=E / 2$. 
Le temps $\theta_{1}$ de montée de tension de l'impulsion est fixé par les inductances et les capacités parasites du montage.

Pendant le temps $\tau_{1}$, la durée de l'impulsion choisie par l'utilisateur, la tension décroît avec la constante de temps $\left(R_{\mathrm{int}}+R_{\mathrm{ch}}\right) \cdot C_{2}$. Ayant choisi une grande valeur pour $C_{2}=3 \times 10^{-6} \mathrm{f}$, cette pente est imperceptible $\left(0,6 \times 10^{-3} \mathrm{~s}\right)$.

Lorsqu'on allume $\mathrm{Th}_{2}$, les potentiels en (A) et en (B) tendent vers la tension de polarisation $-e$. (B) ne pouvant devenir négatif, $\mathrm{Th}_{1}$ s'éteint. La capacité $C_{1}$ se charge avec une constante de temps $R_{\text {int }} . C_{1}$ et la tension en (A) remonte vers $E$. En donnant une valeur convenable à $C_{1}\left(0,2 \times 10^{-6} \mathrm{f}\right)$, la tension en $(\mathrm{A})$ ne redevient positive que lorsque $\mathrm{Th}_{1}$ est désionisé.

Le temps de descente $\theta_{2}$ de l'impulsion est fonction lui aussi des inductances et des capacités du montage.

Lorsque $C_{1}$ est chargé, $\mathrm{Th}_{2}$ s'éteint de lui-même.

On allume $\mathrm{Th}_{3}$ au bout d'un temps tel que $\mathrm{Th}_{2}$ soit désionisé. $C_{1}$ se décharge alors et la tension en (C) reprend la valeur $-e(1 \mathrm{kV})$ avec la constante de temps $\left(R_{3} \cdot C_{1}\right) . \mathrm{Th}_{3}$ s'éteint de lui-même et un nouveau cycle peut recommencer.

2.2 EtAGe D'ATTAQUe. - L'étage d'attaque assure la liaison entre l'étage logique de commande et l'étage de puissance. Il transforme les 3 impulsions de niveaux T.T.L. en impulsions capables de déclencher les thyratrons $T h_{1}, \mathrm{Th}_{2}$ et $T h_{3}$.

L'étage d'attaque, qui est représenté sur la figure 3, est constitué par 3 voies. Chacune d'elles attaque un thyratron. Pour $\mathrm{Th}_{1}$ et $\mathrm{Th}_{2}$, les voies sont identiques, chacune étant constituée par 3 transistors RT 3333A mis en cascade et fonctionnant en régime d'avalanche. Un dispositif analogue a été utilisé au C.E.R.N. pour le déclenchement des chambres à étincelle pulsées [3].

Le $1^{\text {er }}$ transistor est relié à un transformateur de liaison de rapport $(0,6: 1)$. Le $3^{\mathrm{e}}$ transistor est relié à un transformateur élévateur $(3: 1)$ dont le secondaire attaque la grille G2 du CX1157. L'impulsion d'attaque au secondaire du transformateur $(3: 1)$ monte en $40 \mathrm{~ns}$ à $350 \mathrm{~V}$ avec un retard par rapport au signal d'entrée de $100 \mathrm{~ns}$, le « jitter " étant très inférieur à $1 \mathrm{~ns}$. Seul le « jitter » intervient dans les variations de la valeur de $\tau_{1}$ de l'impulsion.

La voie attaquant $\mathrm{Th}_{3}$ est constituée par un thyristor TM8010 dont la gâchette est attaquée par un transformateur $(1: 1)$. L'anode du thyristor est reliée à la grille de $\mathrm{Th}_{3}$ par un autre transformateur $(1: 1)$. 2 diodes à avalanche contrôlées $\mathrm{R} 4 \mathrm{HZ}$ sont branchées aux bornes du thyristor pour lui assurer une protection contre des surtensions éventuelles.

Comme les appels de courant sont importants aux temps de commutation de l'étage de puissance, ils risquent de provoquer une déclenche intempestive des transistors à avalanche. Pour éviter ce phénomène, les précautions suivantes ont été prises lors de la réalisation de cet étage :

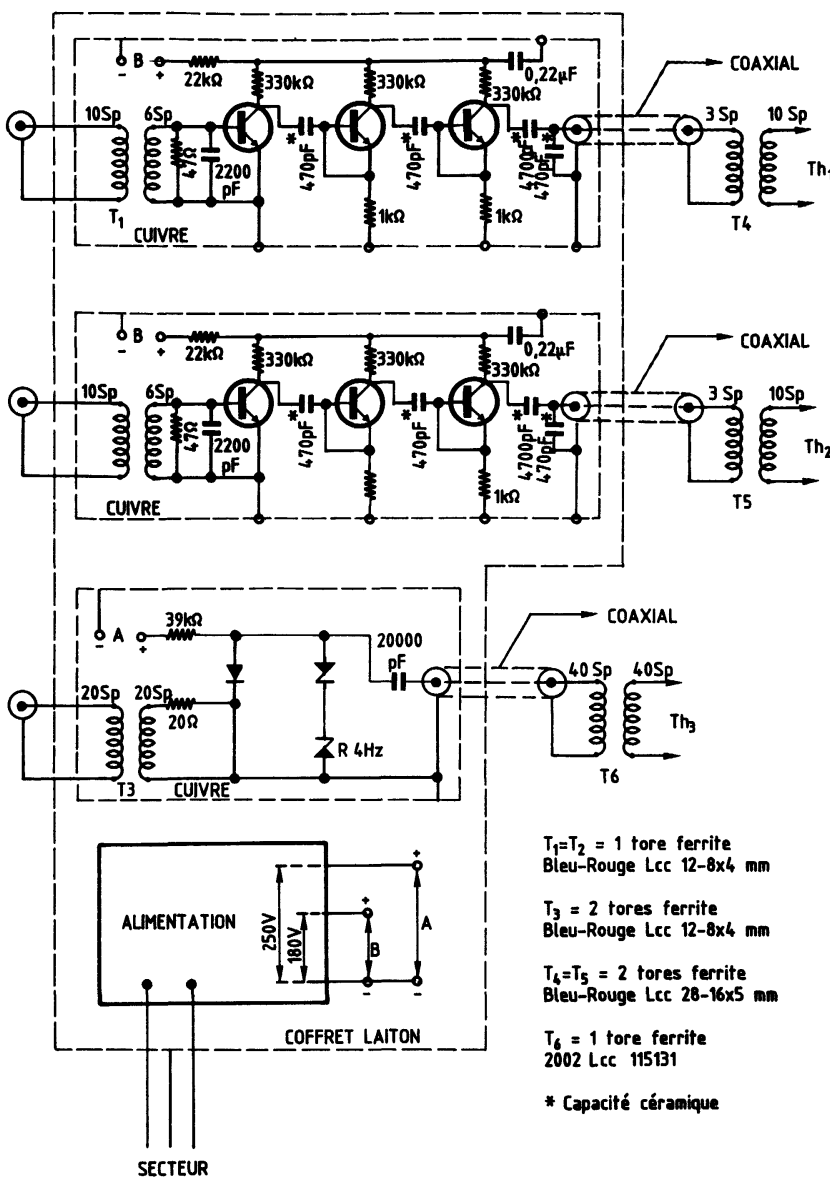

Fig. 3. - Schéma de l'étage d'attaque.

[Scheme of the intermediate stage.]

- les 3 voies sont câblées chacune sur un châssis indépendant en cuivre isolé et blindé par rapport aux 2 autres;

- l'ensemble des 3 voies et leur alimentation ont été placés dans un coffret en laiton pour les blinder vis-àvis de l'extérieur ;

- chaque transformateur de liaison est à tore de ferrite;

- les transformateurs d'entrée sont insérés dans le châssis et ceux de sortie sont placés dans l'étage de puissance;

- les liaisons avec les autres étages (logique et puissance) sont faites par des câbles coaxiaux. Les liaisons pour $\mathrm{Th}_{1}$ et $\mathrm{Th}_{2}$ sont d'égale longueur.

En raison de ce mode de liaison, les potentiels de masse des 3 étages (logique, attaque et puissance) sont indépendants les uns des autres, ce qui atténue les couplages électromagnétiques parasites entre étages.

2.3 ETAGE LOGIQUE. - L'étage de logique ne représentant en fait qu'une forme possible de commande du générateur, nous n'en ferons ici qu'une description succincte.

Son rôle est de créer à chaque déclenchement un train de 3 impulsions nécessaires à l'établissement de 


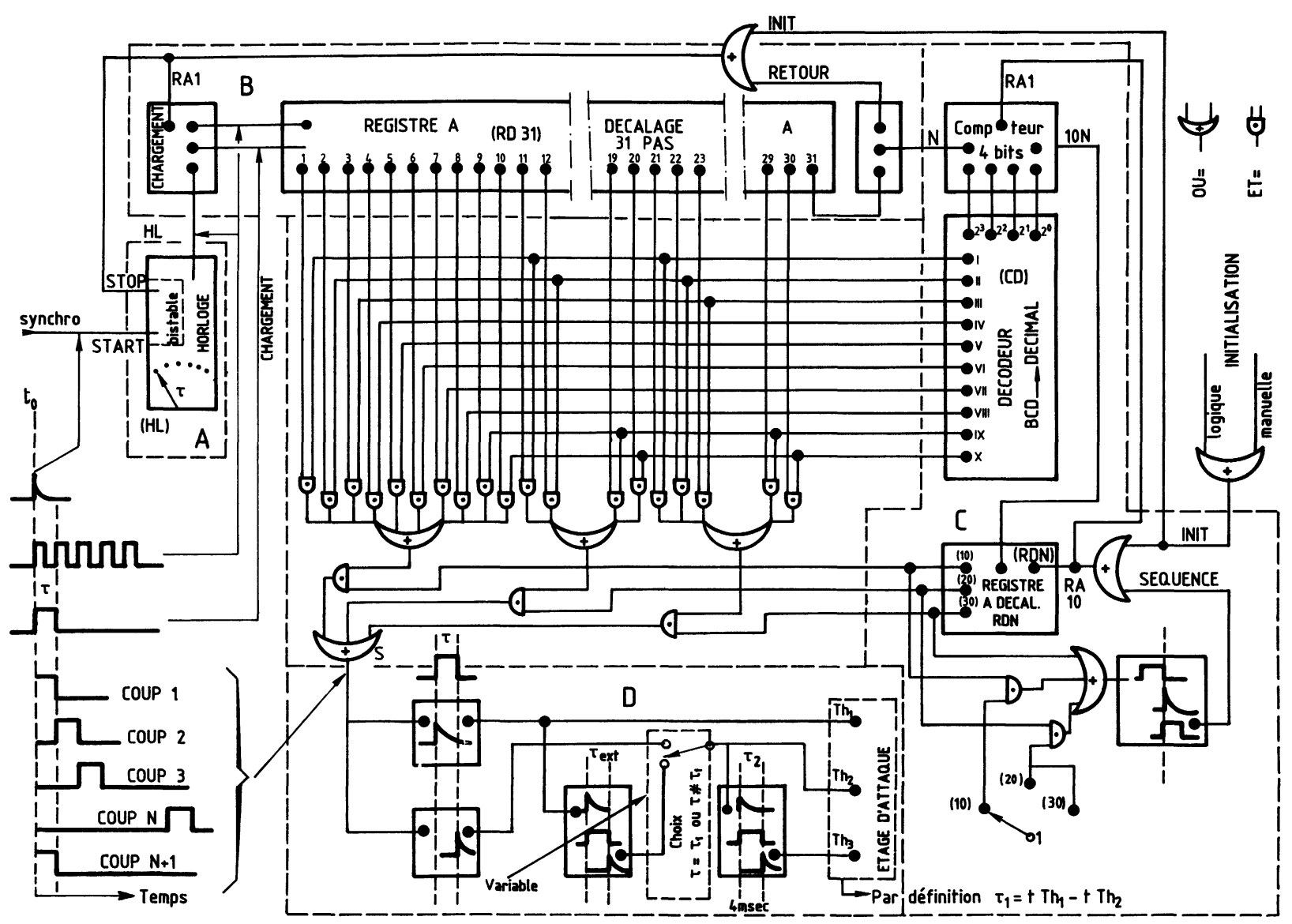

Fig. 4. - Schéma de principe et diagramme de l'étage logique.

[Schematic and working diagrams of the logic stage.]

l'impulsion haute tension et de réaliser les retards séquentiels $n \tau$ par rapport à $t_{0}$ (temps d'excitation par faisceau laser).

Cet étage a été réalisé en circuits intégrés logiques T.T.L. série $S$.

Le câblage est implanté sur des plaquettes de circuits imprimés double face dont l'une est à potentiel fixe (masse). Les plaquettes sont reliées entre elles par un fond de bac en C.I. de même type pour diminuer les couplages parasites. L'ensemble est logé dans un châssis blindé.

A titre documentaire, nous avons représenté le schéma de principe de cet étage sur la figure 4. Il comporte quatre parties :

A : Une horloge "Start-Stop " pilotée par ligne à retard assurant le pas de base $\tau$.

B : Un registre à décalage 31 pas, son décodeur 1-2-4-8 et un réseau logique cablé 30 colonnes 10 lignes assurant le retard séquentiel modulo $N$.

$\mathrm{C}:$ Un registre à décalage (RDN) et un commutateur permettant de choisir pour $N$ la valeur 10,20 ou 30 .

$\mathrm{D}$ : Une série de monostables assurant les séquences temporelles nécessaires à l'allumage et à l'extinction des thyratrons et permettant de choisir $\tau=\tau_{1}$ ou $\tau \# \tau_{1}$.
3. Résultats. - 3.1 L'IMPULSION DE HAUTE TENSION. - La figure 5 représente la photographie de l'impulsion de haute tension délivrée par le généra-

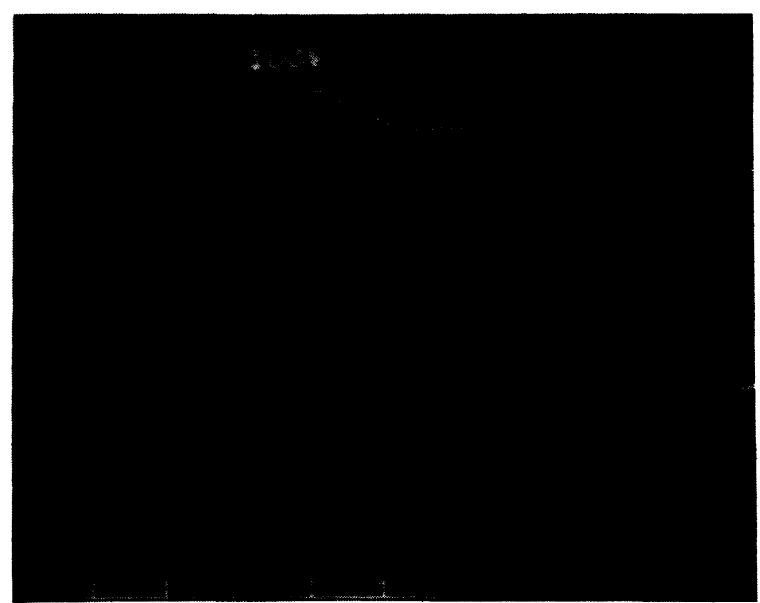

Fig. 5. - Photographie de l'impulsion de haute tension délivrée par le générateur. Echelle verticale : $200 \mathrm{~V} /$ division; échelle horizontale : $20 \mathrm{~ns} /$ division.

[Oscillogram of the high-voltage pulse delivered by the generator. Vertical scale : $200 \mathrm{~V} /$ division; horizontal scale : 20 ns/division.] 
teur pour une amplitude de $1 \mathrm{kV}$ et une durée de $140 \mathrm{~ns}$. Le signal est mesuré par l'intermédiaire d'une sonde associée à un pont diviseur par 2 sur un oscillographe dont la limite supérieure de la bande passante est de $500 \mathrm{MHz}$.

L'ensemble de la chaîne de mesure introduit un allongement du temps de montée de l'impulsion, qui n'est en réalité que de 20 ns de 10 à $90 \%$. Le délai total entre le signal d'attaque $\left(t_{0}\right)$ et la tension de l'impulsion est de 270 ns.

Le « jitter » total est de l'ordre de 2 ns.

Ces résultats ont été obtenus avec un courant de préionisation de $100 \mathrm{~mA}$ pour la grille $G_{1}$ et une polarisation négative de $100 \mathrm{~V}$ de la grille $G_{2}$ des thyratrons CX1157.

L'amplitude des impulsions est limitée à $3 \mathrm{kV}$ actuellement pour les besoins de l'expérience. Compte tenu des caractéristiques des thyratrons, l'amplitude pourrait dépasser la valeur de $10 \mathrm{kV}$.

3.2 APPlication À L'ÉTUde DES ÉTATS DE RYDBERG. - Un exemple typique d'évolution temporelle de la population $N(t)$ d'un niveau de Rydberg obtenue avec le générateur d'impulsions haute-tension pour deux densités différentes de gaz perturbateur est montré figure 6. Chacune des deux courbes est la moyenne sur 100 passages du signal ionique résultant de l'ionisation du niveau $36 \mathrm{~s}$ du rubidium par une impulsion de $920 \mathrm{~V}$, qui est décalée 30 fois d'un pas de 213 ns par rapport à l'excitation laser. Le gaz perturbateur utilisé est le xénon aux densités

$$
N_{\mathbf{X e}}=2,13 \times 10^{12} \mathrm{~cm}^{-3}
$$

et $N_{\mathrm{Xe}}=5,38 \times 10^{12} \mathrm{~cm}^{-3}$. Le logarithme de $N(t)$ varie linéairement en fonction du temps pour chacune des deux densités de xénon. La pente de chaque droite est inversement proportionnelle à la durée de vie du niveau de Rydberg mesurée dans les conditions de l'expérience. Pour $N_{\mathrm{Xe}}=2,13 \times 10^{12} \mathrm{~cm}^{-3}$, la durée de vie mesurée du niveau $36 \mathrm{~s}$ du rubidium vaut $6,66 \mu$ s et pour $N_{\mathrm{Xe}}=5,38 \times 10^{12} \mathrm{~cm}^{-3}$, elle vaut $3,29 \mu \mathrm{s}$. De la variation de sa durée de vie en fonction de la densité de xénon, on déduit la section efficace de dépopulation du niveau par le xénon. Nous avons ainsi mesuré les sections efficaces de dépopulation pour les états ns $(32 \leqslant n \leqslant 45)$ du rubidium par différents gaz rares $(\mathrm{He}, \mathrm{Ne}, \mathrm{Ar}, \mathrm{Xe})$. Les résultats

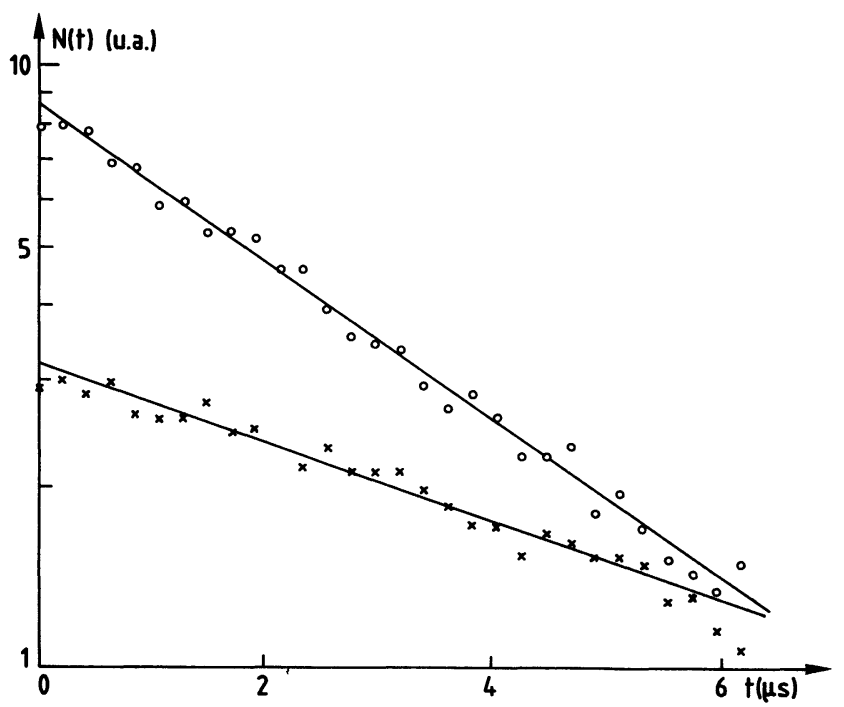

Fig. 6. - Tracé semi-logarithmique de l'évolution temporelle de la population $N(t)$ du niveau $36 \mathrm{~S}$ du rubidium obtenue avec le générateur d'impulsions haute-tension pour deux densités $N_{\mathrm{Xe}}$ de xénon $:(\times): N_{\mathrm{Xe}}=2,13 \times 10^{12} \mathrm{~cm}^{-3}$; (O) : $N_{\mathrm{Xe}}=5,38 \times 10^{12} \mathrm{~cm}^{-3}$. Chacune des deux courbes est ajustée par une droite dont les constantes sont calculées par la méthode des moindres carrés.

[Semi-logarithmic plot of the time-evolution of the population $N(t)$ of the rubidium $36 \mathrm{~S}$ state obtained with the highvoltage pulse generator for two xenon densities $N_{\mathbf{X e}_{\mathrm{e}}}:(\times)$ : $N_{\mathrm{Xe}}=2.13 \times 10^{12} \mathrm{~cm}^{-3} ;(0): N_{\mathrm{Xe}}=5.38 \times 10^{12} \mathrm{~cm}^{-3}$. Both curves are fitted by a straight line, the equation of which is determined by the least-square procedure.]

avec l'hélium sont déjà publiés [1] et les autres feront l'objet d'une publication prochaine.

4. Conclusion. - Les caractéristiques de ce générateur (temps de montée de l'impulsion, délai, faible « jitter ») sont particulièrement bien adaptées à l'étude des niveaux de Rydberg (ionisation par effet Stark, durée de vie, collisions). Le temps de vie des thyratrons devrait permettre l'utilisation du générateur pendant plusieurs années sans difficulté notable. Des modifications de l'étage de puissance sont en cours (impulsions en forme de rampes linéaires variables en temps et en amplitude) pour permettre de nouvelles expériences sur les états de Rydberg. Le générateur aura alors une très grande souplesse d'emploi pour ce type d'étude.

\section{Bibliographie}

[1] Hugon, M., Fournier, P. R. and de Prunelé, E., J. Phys. B : Atom. Mol. Phys. 14 (1981) 4041.

[2] AHRWEILleR, M., Nucl. Instrum. Methods 96 (1971) 205.

[3] FrIEND, B., Nucl. Instrum. Methods 65 (1968) 311. 